\title{
O Uso da Robótica como Apoio à Alfabetização e à Introdução do Pensamento Computacional para Crianças
}

\author{
Poliana Nascimento Ferreira ${ }^{1}$, Aline Mendonça Cordeiro ${ }^{1}$, \\ Tamiris G. S. Lira ${ }^{1}$, Alessandra Sueli Carlos ${ }^{1}$, Carla Lopes Rodriguez ${ }^{1}$ \\ ${ }^{1}$ Centro de Matemática, Computação e Cognição - Universidade Federal do ABC \\ Avenida dos Estados, 5001 - Bairro Santa Terezinha, Santo André, SP, Brazil - CEP: \\ 09210-580 \\ \{poliana.ferreira, alessandra.carlos, c.rodriguez\}@ufabc.edu.br, \\ tamiris.liradaluno.ufabc.edu.br, aline.cordeiro5detec.sp.gov.br
}

\begin{abstract}
This article describes a pedagogical proposal to encourage the development of computational thinking and the use of robotics as a tool to support children's literacy. To do so, here we present the TAAP - Tapete Alfabético, a low cost robotic mat, and two proposals on how to use it. The first one, referring to the use of robotics as a device that supports literacy teachers, shows the potential of using the TAAP in dictation activities and the second one indicates the possibility of stimulating computational thinking in older children through the assembly of the tool.
\end{abstract}

Resumo. Este artigo descreve uma proposta pedagógica para o incentivo ao desenvolvimento do pensamento computacional e o uso da robótica como ferramenta de apoio à alfabetização de crianças. Para tanto, aqui apresentamos o TAAP - Tapete Alfabético, um artefato robótico de baixo custo, e duas propostas pedagógicas de como usá-lo. A primeira, referente ao uso da robótica como dispositivo de apoio a professores alfabetizadores, demonstra o potencial do uso do TAAP em atividades de ditado e diagnóstico, e a segunda indica a possibilidade de estímulo do pensamento computacional em crianças de séries finais do ensino básico por meio da montagem da ferramenta.

\section{Introdução}

A inovação tecnológica, a qual a humanidade vivencia, vem alterando aspectos fundamentais da nossa sociedade como o mundo do trabalho, da saúde, do consumo, dentre outros. O campo educacional certamente não poderia ficar de fora de toda essa transformação. Contudo, os avanços no campo educacional e o uso das Tecnologias da informação e comunicação (TIC) na educação nem sempre acontecem na velocidade que a sociedade anseia. As discussões sobre essas tecnologias como parte do processo das mudanças na sociedade e seus impactos educacionais ainda não têm recebido a devida atenção. Se a maioria dos lares possuem computadores e/ou celulares, se as crianças já nascem e crescem manuseando equipamentos touchscreen, por que ainda assistimos a escola caminhando muito lentamente nessa direção? [Zanella and Lima 2017].

Para a educação acompanhar os avanços tecnológicos, suas ações devem fazer uso dos recursos que facilitem e favoreçam o pensar, o aprender e o criar [Caperton 2010], demonstrando assim a necessidade do ensino de computação como um Saber desde o 
VIII Congresso Brasileiro de Informática na Educação (CBIE 2019)

Anais dos Workshops do VIII Congresso Brasileiro de Informática na Educação (WCBIE 2019)

ensino básico. Uma das maneiras de facilitar o pensar e o aprender é, por exemplo, introduzir às crianças o pensamento computacional para a resolução de problemas. Até a metade do presente século (XXI), todos os seres humanos terão que possuir habilidades relacionadas ao pensamento computacional [Wing 2012]. O pensamento computacional baseia-se no poder e limites de processos computacionais, sejam eles executados por um humano ou por uma máquina, e é uma habilidade fundamental não somente para cientistas de dados, devendo ser utilizado inclusive por crianças do ensino básico na resolução de problemas de leitura, escrita ou aritmética [Wing 2006]. Sendo assim, torna-se tarefa primordial e urgente incluir os princípios do pensamento computacional ainda na fase de alfabetização, projetando para o futuro uma geração que utilize tais princípios em seu raciocínio para a resolução de problemas, sejam eles simples ou mais complexos.

Algumas iniciativas já buscaram a introdução de recursos tecnológicos na educação básica. O Projeto RoPE [Raabe et al.. 2017], por exemplo, usa um brinquedo de programar para ensinar conceitos do pensamento computacional para crianças de 4 a 8 anos. Já no campo da Robótica, [Puziski 2017], por exemplo, utilizou kits de robótica para a construção de uma calculadora em sala de aula e [de Andrade et al.. 2018], por sua vez, propuseram a construção de um teclado musical a partir de materiais reutilizáveis e software livre. Ambos os trabalhos concordam que o uso da robótica foi benéfico e despertou curiosidade e interesse nos alunos, que puderam trabalhar diversos conceitos de forma prática. O trabalho de [de Andrade et al.. 2018], em especial, seguiu princípios da Robótica Livre [Mill and César 2009], um projeto que busca democratizar o ensino da robótica pedagógica. Por acreditarem que os tradicionais kits de robótica trazem limitações relativas ao custo e à impossibilidade de inovar nas criações feitas utilizandoos (afinal, são kits prontos com um manual que indica exatamente os passos que o aluno deve seguir para que consiga montar a ferramenta proposta por eles), os autores propõem o uso de softwares livres e materiais reciclados para o ensino da robótica.

O presente estudo propõe, portanto, a utilização das TIC no processo de alfabetização de crianças e introdução ao pensamento computacional por meio da utilização de uma ferramenta denominada TAAP, que consiste em um tapete projetado com princípios de robótica, utilizando Arduino e materiais recicláveis como papelão, papel e alumínio, acoplado a uma interface de simples manuseio. Assim, partindo do pressuposto que é possível utilizar o ditado como forma avaliativa e de diagnóstico de desenvolvimento de escrita de crianças [Sargiani and Albuquerque 2016], aqui propomos um meio lúdico de integrar pensamento computacional e robótica de modo a auxiliar professores alfabetizadores na apresentação e identificação de palavras dentro de um campo semântico, despertando nos aprendizes a hipótese de escrita proposta por [Ferreiro et al.. 1986], bem como a exploração dos conceitos do pensamento computacional de maneira transversal em temas da Base Nacional Curricular como propõe [Valente 2016], na montagem e construção do TAAP por crianças de nove a dez anos do ensino fundamental e também através de atividades colaborativas de desenvolvimento de instruções para alunos ainda no ensino básico.

\section{TAAP - Tapete Alfabético}

O TAAP - Tapete Alfabético foi desenvolvido (inicialmente na forma de um protótipo) com o objetivo de auxiliar na alfabetização de crianças. Dessa forma, é composto, basicamente, por dois elementos: um tapete que possui "botões" que correspondem cada letra 
VIII Congresso Brasileiro de Informática na Educação (CBIE 2019)

Anais dos Workshops do VIII Congresso Brasileiro de Informática na Educação (WCBIE 2019)

do alfabeto e um software que permite que a interação com o computador.

Para montagem do tapete, a primeira etapa é cortar o papel alumínio no formato, quantidade e tamanho desejados e colar o durex na parte de trás para proteção; a segunda etapa é cortar pequenos pedaços de fita de cobre, colando-os no papel alumínio de modo que haja contato entre o papel e a parte de fora da fita; a terceira etapa é soldar os fios na parte exposta da fita de cobre; a quarta é ligar os fios aos resistores e Arduino (microcontrolador escolhido); a quinta é prender o que foi feito ao papelão, formando a estrutura completa (Figura 1).

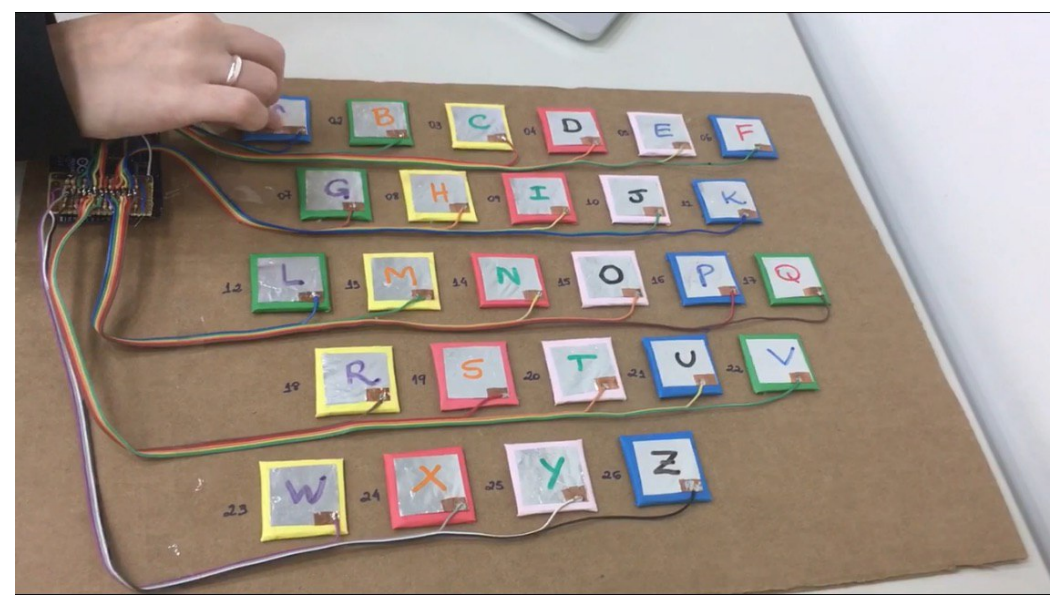

Figura 1. Protótipo do TAAP - Tapete Alfabético

O Arduino é conectado a um computador, o qual alimenta o microcontrolador e interage por meio da porta serial recebendo informações. O software é composto por duas partes: o do microcontrolador e o do computador.

A programação do microcontrolador Arduino é realizada na sua IDE própria, em linguagem C++ adaptada e são utilizadas as bibliotecas: Capacitive Sensor para leitura dos sensores; Arduino Json para o envio das informações dos sensores para a interface do computador. É verificado se algum (e qual) dos sensores está sendo ativado, o índice desse sensor é enviado via serial. A programação da interface no computador é realizada com a linguagem JavaScript, CSS e HTML e o interpretador server-side de código aberto Node.js. Foi construída uma aplicação que é executada localmente após instalação das dependências, as quais são: Serial Port, para a comunicação com o Arduino, e Socket.io, para monitorar a porta e alterar as informações na tela automaticamente. O uso da aplicação é condicionado ao registro da correspondência entre letra e sensor, que pode ser alterado a qualquer momento (Figura 2) e ao cadastro das palavras a serem utilizadas na atividade (Figura 3). Assim, quando um sensor é ativado, a letra correspondente a ele aparecerá na tela do computador, conforme demonstrado (Figura 4).

O hardware do projeto é constituído de materiais recicláveis e de baixo custo, com 26 sensores e dimensão de cada um dos botões de $3 \times 3 \mathrm{~cm}$. Para uma versão full-size do tapete, deve-se aumentar as quantidades de fio, cartolina, papel alumínio e Durex. Além disso, o item de maior valor, o Arduino Mega, pode ser substituído pelos Arduino Nano ou Uno, mais baratos, mas com menos portas. A (Tabela 1) lista os valores dos materiais utilizados para a construção do protótipo. 
VIII Congresso Brasileiro de Informática na Educação (CBIE 2019)

Anais dos Workshops do VIII Congresso Brasileiro de Informática na Educação (WCBIE 2019)

\section{TAAP}

Bem vindo ao TAAP, primeiro escreva qual letra corresponde a cada posição

na ferramenta

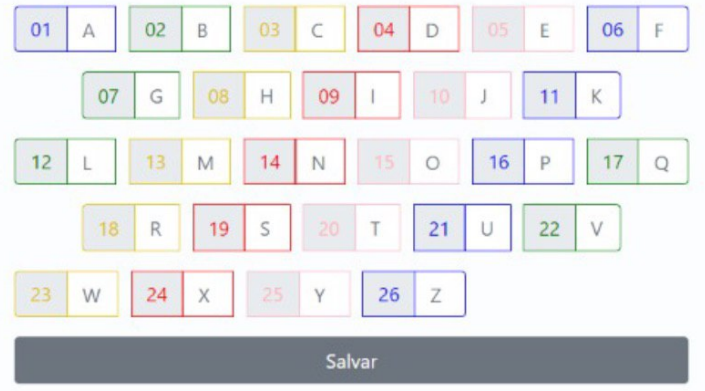

Figura 2. Registro de correspondência entre letra e sensor

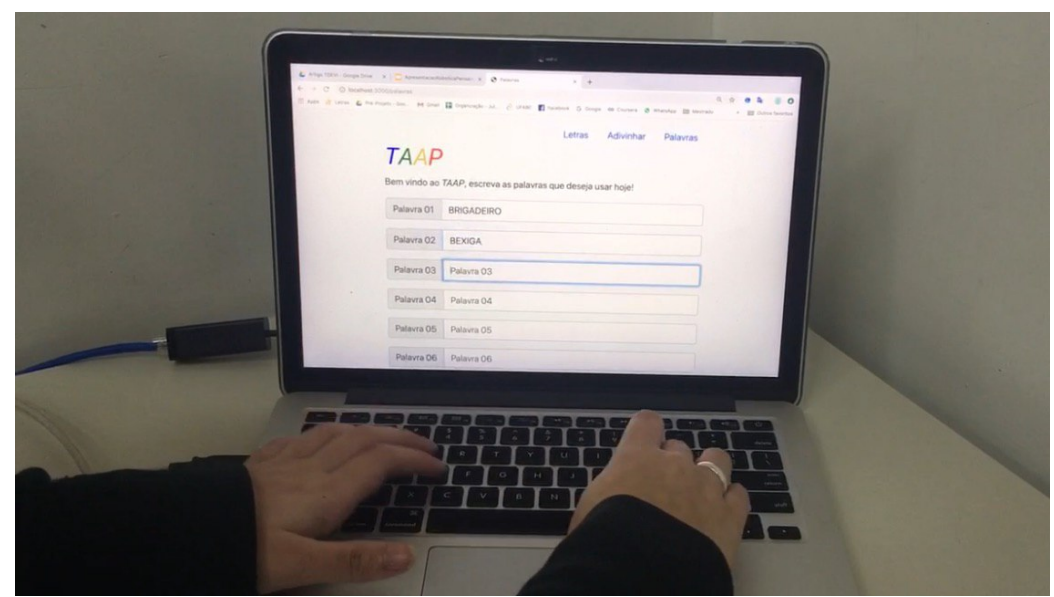

Figura 3. Cadastro de palavras

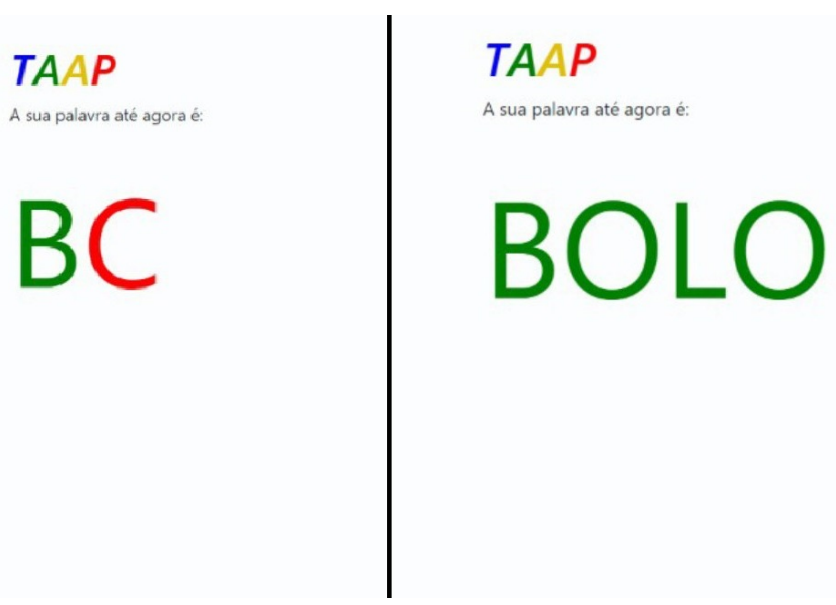

Figura 4. Exemplo de funcionamento da ferramenta com a palavra "BOLO" 
VIII Congresso Brasileiro de Informática na Educação (CBIE 2019)

Anais dos Workshops do VIII Congresso Brasileiro de Informática na Educação (WCBIE 2019)

Tabela 1. Valores do protótipo construído

\begin{tabular}{|c|c|c|l|}
\hline Quantidade & Descrição & Valor unitário & Valor total \\
\hline 26 un & Resistor 10k & $\mathrm{R} \$ 0,07$ & $\mathrm{R} \$ 1,82$ \\
\hline 26 un & Pin Head & 40 un $=\mathrm{R} \$ 0,42$ & $\mathrm{R} \$ 0,27$ \\
\hline $8 \mathrm{~m}$ & Fio & $420 \mathrm{~m}=\mathrm{R} \$ 110,00$ & $\mathrm{R} \$ 2,09$ \\
\hline $0,5 \mathrm{~m}$ & Fita de cobre & $30 \mathrm{~m}=\mathrm{R} \$ 74,96$ & $\mathrm{R} \$ 1,24$ \\
\hline $1,25 \mathrm{un}$ & Cartolina & $10 \mathrm{un}=\mathrm{R} \$ 7,20$ & $\mathrm{R} \$ 0,90$ \\
\hline $0,1 \mathrm{~m}$ & Rolo de papel alumínio & $30 \mathrm{~m}=\mathrm{R} \$ 9,49$ & $\mathrm{R} \$ 0,32$ \\
\hline $0,8 \mathrm{~m}$ & Durex & $40 \mathrm{~m}=\mathrm{R} \$ 3,08$ & $\mathrm{R} \$ 0,62$ \\
\hline & Papelão & $\mathrm{R} \$ 0,00$ & $\mathrm{R} \$ 0,00$ \\
\hline 1 & Arduino Mega & $\mathrm{R} \$ 89,90$ & $\mathrm{R} \$ 89,90$ \\
\hline & & & $\mathbf{R \$ 9 7 , 1 6}$ \\
\hline
\end{tabular}

\section{Proposta Pedagógica}

O TAAP foi idealizado com a intenção de ser utilizado como ferramenta de auxílio a professores em sala de aula. Dessa maneira, a seguir são propostas duas possibilidades de utilização da ferramenta: a primeira com foco no uso do TAAP para apoio à alfabetização, e a segunda com foco no uso da ferramenta para o desenvolvimento do pensamento computacional.

\subsection{Alfabetização}

O trabalho foi iniciado com pesquisas bibliográficas que relatam diferentes metodologias e sequências didáticas utilizadas em turmas de alfabetização, com crianças de 6 a 7 anos nas escolas públicas da cidade de São Paulo. Foi realizado um estudo empírico informal com professores que possuem grande experiência na alfabetização de crianças baseados no programa Ler e Escrever, implantado na prefeitura de São Paulo em 2006. O programa visa a alfabetização de todas as crianças nas três séries iniciais do ensino fundamental, utilizando a teoria proposta por [Ferreiro et al.. 1986], que busca proporcionar à criança, em substituição à mecanização dos métodos de alfabetização tradicionais, a construção de seu próprio conhecimento, formulando suas hipóteses de leitura e escrita.

Para utilizar o TAAP em uma aula de alfabetização, o primeiro passo é realizar o planejamento da aula, definindo um tema e selecionando o campo semântico que será trabalhado pelo professor alfabetizador, o qual consiste em uma lista de palavras (vocabulário) relacionadas ao tema, classificadas em monossílabas, dissílabas, trissílabas e polissílabas, além de uma frase que contenha palavras do campo semântico escolhido.

O TAAP foi projetado para ser uma ferramenta adaptável às necessidades da aula pelo professor. Sendo assim, o professor poderá escrever as letras e símbolos na interface do papel alumínio e configurar a posição dos símbolos pela interface acoplada ao computador. A face superior do TAAP, onde a criança irá tocar/pisar, é feita de papel alumínio e apagável se utilizada a caneta apropriada, tornando-a reutilizável para outras finalidades.

As palavras devem, então, ser armazenadas no TAAP pelo professor por meio de uma interface amigável e colorida, buscando facilitar a interação entre o professor alfabetizador e a ferramenta. $\mathrm{O}$ ambiente para a aula deve ser intencionalmente organizado, 
VIII Congresso Brasileiro de Informática na Educação (CBIE 2019)

Anais dos Workshops do VIII Congresso Brasileiro de Informática na Educação (WCBIE 2019)

contendo uma lista fixa de palavras que são trabalhadas todos os dias com os aprendizes (por exemplo com os nomes dos alunos) e o alfabeto, que será substituído pelo TAAP.

As imagens relacionadas ao campo semântico escolhido pelo professor alfabetizador são apresentadas aos alunos sem, no entanto, demonstrar a escrita. As palavras são então introduzidas oralmente em histórias, contos e frases pelo professor alfabetizador, que provoca, estimula e questiona os alunos para que eles indiquem no TAAP as letras que iniciam as palavras, as letras com que terminam as palavras e as letras que diferenciam uma palavra de outra. Ao acertar a letra questionada pelo professor, o TAAP mostrará a letra digitada na interface, exibindo-a na cor verde em caso de acerto. Ao errar a palavra, a letra digitada será exibida em vermelho e o professor alfabetizador poderá indicar a letra correta ou ainda recomeçar o processo questionando novamente os alunos e mostrando a diferença entre uma palavra e outra utilizando o computador acoplado ao TAAP.

Podem ainda ser propostas palavras relacionadas ao grupo semântico para que os alunos percorram o tapete TAAP indicando a escrita, jogos com palavras e desafios em que os alunos poderão participar de forma individual ou em grupos. O tapete pode também, a critério do professor, ser utilizado para realizar o diagnóstico das crianças, como ferramenta de apoio para ajudar o alfabetizador a descobrir a fase de alfabetização na qual as crianças se encontram.

\subsection{Pensamento Computacional}

Os nativos digitais possuem facilidade em interagir com os produtos eletrônicos, o que não significa que possuem habilidades para produzir e criar as tecnologias, mas apenas consumi-las. Desenvolver o pensamento computacional é a competência básica para produção e criação de tecnologias.

"O Pensamento Computacional é uma distinta capacidade criativa, crítica e estratégica humana de saber utilizar os fundamentos da Computação nas mais diversas áreas do conhecimento, com a finalidade de identificar e resolver problemas colaborativamente através de passos claros de tal forma que uma pessoa ou uma máquina possam executálos eficazmente" [Brackmann 2017], é uma forma de pensar e organizar ideias ao qual incluem um conjunto de habilidades que ajudam na resolução de problemas. Propicia desenvolvimento do homem como cidadão e é necessário para formação de um bom profissional pois envolve programação, raciocínio lógico, trabalho em equipe, resolução de problemas, empreendedorismo e criatividade.

Um estudo do professor Christian Brackmann [Brackmann 2017] mostra que a idade de sete anos é a ideal para introduzir o desenvolvimento desta competência nas crianças, já que possibilita a resolução de problemas do cotidiano de maneira mais organizada. O importante é mostrar para a criança que o computador não pensa sozinho, quem precisa raciocinar são as pessoas que fazem parte das equipes que desenvolvem as tecnologias, e que o robô apenas executa as instruções dadas.

Existem quatro etapas para organização do pensamento computacional. A primeira etapa, decomposição, consiste em dividir o problema em problemas menores, portanto mais fáceis. Na segunda etapa, padrões, deve-se identificar os padrões que geram o problema. A terceira etapa, abstração, os fatores de geração do problema são reconhecidos e deve-se ignorar os detalhes de uma solução de modo que ela possa ser válida para 
VIII Congresso Brasileiro de Informática na Educação (CBIE 2019)

Anais dos Workshops do VIII Congresso Brasileiro de Informática na Educação (WCBIE 2019)

diversos problemas. Por fim, a quarta etapa, algoritmo, consiste em propor uma ordem ou sequência de passos para resolver o problema [Brackmann 2017]

A importância da introdução de princípios de programação e criação de soluções computacionais por alunos do Ensino Fundamental tem sido uma iniciativa em vários países como os Estados Unidos, que lançou o manifesto Computer Science is for Everyone [White House 2013] que propõe o ensino de princípios de programação e criação de tecnologias desde o ensino fundamental, e o Reino Unido, que substituiu a disciplina de Informática, onde os alunos apenas aprendiam a operar computadores com softwares de escritório, por Computação, que está fundamentada no tripé Ciência da Computação, Tecnologia da Informação e Letramento Digital [Department for Education 2013]. No Brasil, a Base Nacional Comum Curricular [Ministério da Educação 2018] determina algumas diretrizes para o desenvolvimento do pensamento computacional já no ensino fundamental. No entanto, essas diretrizes aparecem incorporadas em unidades temáticas da matemática e, muitas vezes, de forma equivocada, como apontado na Nota Técnica da Sociedade Brasileira de Computação sobre a BNCC-EF e BNCC-EM [Sociedade Brasileira de Computação 2018]. Aqui, são propostas duas maneiras de utilização do TAAP para introdução ao P.C. na educação básica: uma na montagem da ferramenta e a outra uma atividade utilizando o tapete em si.

A primeira proposta é que os alunos do ensino fundamental sejam capazes de construir a ferramenta com princípios de Ciência da Computação. Na etapa de decomposição, o TAAP deverá ser decomposto, dividindo sua estrutura na construção do tapete (teclado), esquema eletroeletrônico e software que será utilizado na interface acoplada ao TAAP. Os alunos, orquestrados pelo(a) professor(a), irão primeiramente construir o tapete, cortando o papelão, revestindo-o com papel alumínio e anexando a fita de cobre a cada tecla do tapete. Na sequência, o(a) professor(a) irá introduzir os princípios eletroeletrônicos, demonstrando como interligar cada tecla ao arduíno por meio de fios soldados às teclas, bem como a conexão do arduino ao computador pela porta USB. $\mathrm{O}$ software para o funcionamento do TAAP pode ser desenvolvido pelos alunos para aplicações variadas em uma etapa futura, mas também há a possibilidade de instalar o pacote de software disponibilizado pela equipe.

$\mathrm{Na}$ etapa de padronização, deve-se apresentar aos alunos outros exemplos de utilização do Arduíno na resolução de problemas, para que sejam capazes de identificar que, independente da solução para a qual o arduíno será empregado, o padrão de conexão obedece aos mesmos princípios. Na etapa de abstração, o professor deverá apresentar diferentes formas de utilização da ferramenta, como por exemplo utilizá-lo como teclado numérico no ensino de operações matemáticas simples, na formação de sílabas, letras, palavras ou até mesmo frases, bem como utilizar o teclado em jogos que envolvam palavras. $\mathrm{O}(\mathrm{A})$ professor(a) deverá ainda provocar os alunos que estão realizando a montagem da ferramenta, convidando-os a pensar situações em que eles poderiam utilizar o teclado TAAP. Todas as sugestões propostas pelos alunos devem ser consideradas e anotadas, para que futuramente possam ser, havendo viabilidade, implementadas e testadas. Por fim, a quarta etapa, algoritmo, consiste em propor a criação de um manual de instruções para montagem, teste e utilização do TAAP, seguindo as ações que os próprios alunos executaram. O algoritmo proposto pelos alunos deve então ser validado por um outro grupo que fará a montagem do TAAP, para que possíveis correções possam ser implementadas, 
VIII Congresso Brasileiro de Informática na Educação (CBIE 2019)

Anais dos Workshops do VIII Congresso Brasileiro de Informática na Educação (WCBIE 2019)

gerando assim uma nova versão do manual.

A segunda proposta é uma atividade dinâmica para crianças ainda na educação básica e é descrita a seguir. Um mapa contendo a posição de cada letra (correspondente à forma que elas foram registradas no sistema) deverá ser confeccionado pelo professor, que não deixará nenhuma letra escrita no tapete. Este mapa deverá ser entregue a uma criança que, ao verificar a posição de cada letra no mapa, deverá dar instruções para que uma outra criança chegue até essa letra e possa pressioná-la até que a palavra ditada pelo professor possa ser completada corretamente. As instruções, do tipo "dê um passo para a esquerda", "dê um passo para frente", "dê um passo para trás", "dê um passo para a direita", "pressione o botão", poderão tanto ser dadas verbalmente quanto com a utilização de cartões de instruções, como os AlgoCards [Brackmann 2017].

Esperamos que com atividades desse tipo os alunos possam exercitar e entender o conceito de todas as etapas de organização do pensamento computacional. Os alunos deverão notar que, para conseguir resolver o problema de "acertar a palavra", deverão, antes, resolver o problema de "acertar a letra" para cada letra presente na palavra, desenvolvendo, assim, conceitos de decomposição. Deverão perceber, também, que o conjunto de instruções para acertar uma letra da palavra deverá ser sempre o mesmo quando ela se repetir (quando o mapa, obviamente, não mudar), o que exercita a etapa dois do pensamento computacional, padrões, quando eles são identificados. Ao entenderem que, independente da palavra sugerida pelo professor, precisarão seguir um conjunto de instruções para solucionar o problema de "acertar a palavra" e, mais do que isso, perceber que cada palavra possui um conjunto diferente de letras e para "acertar a palavra" precisarão "acertar a letra" várias vezes, os alunos praticarão a abstração. Por fim, o exercício da última etapa, algoritmo, se dá com a percepção de que, para que cada palavra seja construída, um conjunto de instruções, definido pelos próprios alunos, deverá ser seguido.

Além do exercício das etapas do pensamento computacional, também esperamos que a aplicação dessa atividade possa incentivar a colaboração e o trabalho em equipe entre os alunos, já que a criança que pressionará a tecla correspondente à letra somente conseguirá o fazer caso siga corretamente as instruções dadas por seus colegas. Também é possível determinar papéis para os alunos, de forma que a criança que realiza as instruções possa assumir o papel de "computador" e, quem dá as instruções, o "programador" e que eles entendam, de forma lúdica, que o "computador" só poderá acertar se o "programador" for claro e der as instruções corretas para que ele realize a tarefa desejada.

\section{Resultados Preliminares e Perspectivas Futuras}

Até o momento, um protótipo funcional e de baixo custo da ferramenta foi construído, com um tapete em tamanho reduzido e um software simples que se comunica com ele. A partir deste protótipo, foi possível identificar seu potencial como dispositivo de auxílio à alfabetização e à introdução do pensamento computacional para crianças. Este, no entanto, é um estudo preliminar e, portanto, o TAAP ainda não foi suficientemente explorado e continuará a ser trabalhado pelas autoras no futuro.

Em relação à ferramenta e à interface, os próximos passos são a construção do tapete em tamanho real, modularizar a programação do Arduino para funcionar com qualquer número de sensores e criar a interface para a atividade de pensamento computacional. Além disso, o programa será transformado em aplicação Desktop de fácil 
VIII Congresso Brasileiro de Informática na Educação (CBIE 2019)

Anais dos Workshops do VIII Congresso Brasileiro de Informática na Educação (WCBIE 2019)

instalação com o Framework Electron (a integração entre ele e o Serial Port ainda está sendo feita pelas desenvolvedoras). Pretende-se, também, testar e validar a ferramenta e as propostas pedagógicas em escolas parceiras e disponibilizar gratuitamente o passo a passo de construção, o software e as estratégias de uso que foram apresentadas nesse trabalho, além de verificar a possibilidade de expansão do uso da ferramenta em outras áreas e por diferentes faixas etárias. Os tutoriais e manuais do TAAP serão facilitados aos professores, para que permitam aos mesmos a construção da ferramenta com os alunos de quarto e quinto anos do ensino fundamental.

\section{Conclusão}

A utilização da robótica como ferramenta para melhorar a interação e a comunicação de crianças não é uma novidade. [Silva 2012] afirma que a presença de robôs pode ser uma estratégia motivacional para algumas crianças, maximizando as suas interações e/ou potencializando a aprendizagem de novas competências. O desenvolvimento tecnológico exponencial, em especial o computacional, e no campo da robótica da sociedade atual, impulsiona sua inserção em todas as áreas, inclusive e de forma emergencial, na educacional. Torna-se então imprescindível a manipulação e inserção de tais recursos como ferramentas para o processo de ensino e aprendizagem [de Souza et al.. 2016].

Democratizar a tecnologia, propondo soluções de baixo custo e de fácil desenvolvimento e aquisição parece-nos então ser um dos caminhos para que escolas públicas consigam introduzir a tecnologia para crianças e adolescentes, impulsionando-as não apenas ao consumo de tecnologia, o que para os nativos digitais já parece ser algo comum, como também à construção e desenvolvimento de ferramentas tecnológicas.

Sendo assim, vemos o TAAP como uma possibilidade de inserção de robótica no momento da alfabetização de criança, bem como no desenvolvimento do pensamento computacional, a fim de contribuir para transformar o aluno, que ocupa muitas vezes um papel passivo na utilização de ferramentas computacionais prontas, em um agente ativo na construção e entendimento de ferramentas tecnológicas. Esperamos também que seu uso possa trazer benefícios como o desenvolvimento do raciocínio lógico, o incentivo ao estudo interdisciplinar (por promover a integração de áreas como robótica, eletrônica, pensamento computacional e língua portuguesa), acesso democrático (pela fácil utilização da ferramenta e baixo custo de montagem) e à aproximação dos alunos e professores com a ciência e tecnologia.

\section{Referências}

Brackmann, C. P. (2017). Desenvolvimento do pensamento computacional através de atividades desplugadas na educação básica. Universidade Federal do Rio Grande do Sul (UFRGS), Porto Alegre, RS, Brasil.

Caperton, I. H. (2010). Toward a theory of game-media literacy: Playing and building as reading and writing. International Journal of Gaming and Computer-Mediated Simulations (IJGCMS), 2(1):1-16.

de Andrade, T. G. M., Vicente, Z. R. S., Leite, H. A. L., Cabral, A. P. C., Baldow, R., Rocha, N., and Leão, M. B. C. (2018). A robótica livre e o ensino de física e de programação: desenvolvendo um teclado musical eletrônico/free robotics and the tea- 
VIII Congresso Brasileiro de Informática na Educação (CBIE 2019)

Anais dos Workshops do VIII Congresso Brasileiro de Informática na Educação (WCBIE 2019)

ching of physics and programming: developing an electronic music keyboard. Texto Livre: Linguagem e Tecnologia, 11(3):317-330.

de Souza, I. M. L., da Silva Rodrigues, R., and Andrade, W. (2016). Introduçao do pensamento computacional na formaçao docente para ensino de robótica educacional. In Anais dos Workshops do Congresso Brasileiro de Informática na Educação, volume 5, page 1265.

Department for Education (2013). The national curriculum in england: Framework document. Department for Education London.

Ferreiro, E., Teberosky, A., and Lichtenstein, D. M. (1986). Psicogênese da língua escrita. Artes Médicas.

Mill, D. and César, D. (2009). Robótica pedagógica livre: sobre inclusão sócio-digital e democratização do conhecimento. Perspectiva, 27(1):217-248.

Ministério da Educação (2018). Base nacional comum curricular. http://basenacionalcomum.mec.gov.br, acesso em: Setembro de 2019. Secretaria da Educação Básica, Brasil.

Puziski, M. (2017). Construindo uma calculadora: uma atividade envolvendo robótica, programação e matemática. REMAT, 3(1):136-146.

Raabe, A., Rosário, T., Martins, R., Santana, A. L. M., Souza, F. T., and Silva, R. (2017). Rope-brinquedo de programar e plataforma de aprender. In Anais do Workshop de Informática na Escola, volume 23, page 1119.

Sargiani, R. d. A. and Albuquerque, A. (2016). Análise das estratégias de escrita de crianças pré-escolares em português do brasil. Psicologia Escolar e Educacional, 20(3):591-600.

Silva, S. I. A. d. (2012). Estudo do efeito da utilização de uma plataforma robótica na intervenção em crianças com perturbações do espetro do autismo. $\mathrm{PhD}$ thesis.

Sociedade Brasileira de Computação (2018). Nota Técnica da Sociedade Brasileira de Computação sobre a BNCC-EF e BNCC-EM. https://www.sbc.org.br/institucional3/cartas-abertas/send/93-cartas-abertas/1197-nota-tecnica-sobre-a-bncc-ensinomedio-e-fundamental, acesso em Outubro de 2019.

Valente, J. A. (2016). Integração do pensamento computacional no currículo da educação básica: diferentes estratégias usadas e questões de formação de professores e avaliação do aluno. Revista e-Curriculum, 14(3):864-897.

White House (2013). Computer Science is for Everyone! https://obamawhitehouse.archives.gov/blog/2013/12/11/computer-science-everyone, acesso em Setembro de 2019.

Wing, J. M. (2006). Computational thinking. Communications of the ACM, 49(3):33-35.

Wing, J. M. (2012). Computational thinking. Microsoft Asia Faculty Summit. Tianjin, China.

Zanella, B. R. D. and Lima, M. d. F. W. P. (2017). Refletindo sobre os fatores de resistência no uso das tics nos ambientes escolares. Scientia cum Industria, 5(2):78-89. 